\title{
NU-IN: Nucleotide evolution and input module for the EvolSimulator genome simulation platform
}

Katrina M Dlugosch ${ }^{1 *}$, Michael S Barker ${ }^{2,3}$, Loren H Rieseberg ${ }^{1,3}$

\begin{abstract}
Background: There is increasing demand to test hypotheses that contrast the evolution of genes and gene families among genomes, using simulations that work across these levels of organization. The EvolSimulator program was developed recently to provide a highly flexible platform for forward simulations of amino acid evolution in multiple related lineages of haploid genomes, permitting copy number variation and lateral gene transfer. Synonymous nucleotide evolution is not currently supported, however, and would be highly advantageous for comparisons to full genome, transcriptome, and single nucleotide polymorphism (SNP) datasets. In addition, EvolSimulator creates new genomes for each simulation, and does not allow the input of user-specified sequences and gene family information, limiting the incorporation of further biological realism and/or user manipulations of the data.

Findings: We present modified C++ source code for the EvolSimulator platform, which we provide as the extension module NU-IN. With NU-IN, synonymous and non-synonymous nucleotide evolution is fully implemented, and the user has the ability to use real or previously-simulated sequence data to initiate a simulation of one or more lineages. Gene family membership can be optionally specified, as well as gene retention probabilities that model biased gene retention. We provide PERL scripts to assist the user in deriving this information from previous simulations. We demonstrate the features of NU-IN by simulating genome duplication (polyploidy) in the presence of ongoing copy number variation in an evolving lineage. This example is initiated with real genomic data, and produces output that we analyse directly with existing bioinformatic pipelines.

Conclusions: The NU-IN extension module is a publicly available open source software (GNU GPLv3 license) extension to EvolSimulator. With the NU-IN module, users are now able to simulate both drift and selection at the nucleotide, amino acid, copy number, and gene family levels across sets of related genomes, for user-specified starting sequences and associated parameters. These features can be used to generate simulated genomic datasets under an extremely broad array of conditions, and with a high degree of biological realism.
\end{abstract}

\section{Introduction}

The current explosion of genomic sequence data is generating unprecedented insights into the structure and evolution of genomes. Among the most profound recent discoveries is the extent to which gene copy number variation and the gain and loss of lineage specific duplications are pervasive and ongoing features of evolution in many organisms [1]. Such paralogs are known to affect

\footnotetext{
* Correspondence: katrina.dlugosch@gmail.com

'Department of Botany, University of British Columbia, Vancouver, BC V6T1Z4, Canada

Full list of author information is available at the end of the article
}

phenotypes directly and to play important roles in the evolution of gene functions and divergence among species, e.g. [2-4]. Accordingly, their retention or loss appears to be shaped by selection in some cases [5]. This means that comprehensive studies of genomes must incorporate evolution at the levels of gene and gene family loss and gain, as well as the traditional scales of nucleotide and amino acid mutation. Simulations that integrate across all of these levels to generate different evolutionary scenarios will provide powerful tools for testing hypotheses about how evolution works.

\section{Biomed Central}


The simulation platform EvolSimulator [6] was recently developed to accommodate many of these needs. It generates the coding sequences of an ancestral haploid genome, uses this genome to initiate any number of lineages, and evolves the lineages forward under different selective regimes. Users can specify rates of nucleotide mutation, gene duplication and loss, as well as lateral gene transfer, if desired. EvolSimulator is currently one of the only programs available for performing such detailed genome-scale simulations, and it provides a particularly powerful platform for testing aspects of gene family evolution [7].

Currently, EvolSimulator (as of version 2.1.0) is designed to simulate only amino acid evolution. A nucleotide sequence exists for every genome, and mutations are evaluated at each position according to nucleotide substitution models set by the user, but only nonsynonymous changes are retained and propagated through the simulation. A full implementation of (synonymous and non-synonymous) nucleotide evolution in the simulation would be highly advantageous for testing hypotheses at the nucleotide level, and for comparison to increasingly available genome, transcriptome, and single nucleotide polymorphism (SNP) datasets. Many analyses of sequence and genome evolution utilize patterns of both synonymous and non-synonymous nucleotide changes to infer relationships within and between genomes and distinguish the action of selection [8]. Simulations that include neutral and non-neutral nucleotide evolution are therefore particularly important for evolutionary hypothesis testing.

In addition, particular evolutionary scenarios will often require specific data types, different parameter sets at different time points, and/or user manipulations of the data. These objectives are most easily achieved by allowing users to input their own sequence and other genomic information as the starting material for a given simulation. For example, it is desirable to use data from real organisms in cases where the simulation will be compared directly to real data, or used to test analysis pipelines which are dependent on biological realism (such as protein motifs). It is also commonly helpful to restart a simulation, for instance to provide replication from the same starting genome, multi-stage simulations where different parameter combinations are needed, and manual manipulation of genomes (such as whole genome duplication, see example below) within the simulation. EvolSimulator is not designed to accommodate these inputs at present, and instead generates a new genome as the ancestor for each simulation. We have developed NU-IN, an extension to the EvolSimulator platform to implement nucleotide evolution and offer user data input, along with the existing program features (see Additional File 1: NU-IN Download 1.1.0 for the source code and documentation for this software).

\section{Software Description}

NU-IN takes advantage of EvolSimulator's existing nucleotide-based mutation machinery and implements it fully. EvolSimulator generates nucleotide changes according to user specified rates of mutation and nucleotide bias. Previously, only mutations leading to an amino acid change that survived selection were recorded. With NU-IN, all synonymous (silent) nucleotide mutations as well as the non-synonymous mutations are reported. Synonymous changes are selectively neutral and remain in the genome unless they co-occur with an amino acid change that is eliminated by selection (as we imagine happens in nature).

In addition, NU-IN allows users to start simulations with their own data, including genes from real organisms and/or genomes already generated by EvolSimulator. Input genomes may be any fasta-formatted nucleotide sequences in reading frame. NU-IN processes input data in several steps: First, the genome file is read, the genes are numbered sequentially, and each gene is assigned as the first member of its gene family. Every gene is then assigned a 'usefulness' value in all selective environments ('habitats' and 'niches') to be used in the program. Usefulness values are probabilities of gene retention, dictating how important it is to prevent a gene from being lost in an environment if the genome size happens to shrink (where parameters for genome size variation are set by the user). This represents selection at the level of gene loss. All of these steps mimic the same process used by EvolSimulator2.1.0 when it creates a simulated starting genome.

NU-IN also creates the option for the user to provide their own gene family membership and gene usefulness information. When inputting real data, gene family membership information can be derived from additional analyses of gene relationships in the genome [9]. Gene usefulness could be tied to knowledge about historical patterns of gene retention (such as biased retention of transcription factors [10]). For inputs of genomes generated by previous runs of the simulation, gene family and usefulness information is embedded in the output files. The NU-IN download provides several PERL scripts to aid users in parsing sequence, family, and usefulness information from the simulation output.

\section{Example Usage}

We expect that users will find this to be an exceptionally practical tool with which they can test a very broad array of hypotheses about sequence and genome evolution. For example, simulated datasets can be generated 
to evaluate the impact of mutation rate, divergence times, and/or selection on our ability to detect reticulate evolution (lateral gene transfer or hybridization), copy number variation, or genome duplication in samples of genomic data. To demonstrate one such an approach, we simulated a whole genome duplication (polyploidy) in an evolving lineage with a constant background rate of gene gain and loss. We initiated our simulation with coding sequences from the spike moss Selaginella moellendorffii genome (10 Sep 2008 release [11]), which shows no evidence of recent or ancient polyploidy. We evolved this genome for $5.0 \mathrm{Ks}$ (synonymous substitutions/site) to allow it to stabilize on a constant rate of gene turnover (see Additional File 2: Example Parameters for the parameters used), and produce an age distribution of simulated paralogs that is similar to the original data (Figure 1 inset). Using the output of this simulation, we doubled the genome manually and input this polyploid genome and its gene family and usefulness information back into the simulation to evolve for an additional 0.05 and $0.45 \mathrm{Ks}$.

For the original, simulated, and duplicated genomes, we used an existing bioinformatic pipeline to identify the Ks values for each duplication event in each gene family tree $[12,13]$ (available as 'DupPipe' at EvoPipes. net [14]). We used a random sample of 10,000 genes for these analyses, similar to what is available in many publicly available EST datasets (e.g. [12]). Briefly, this

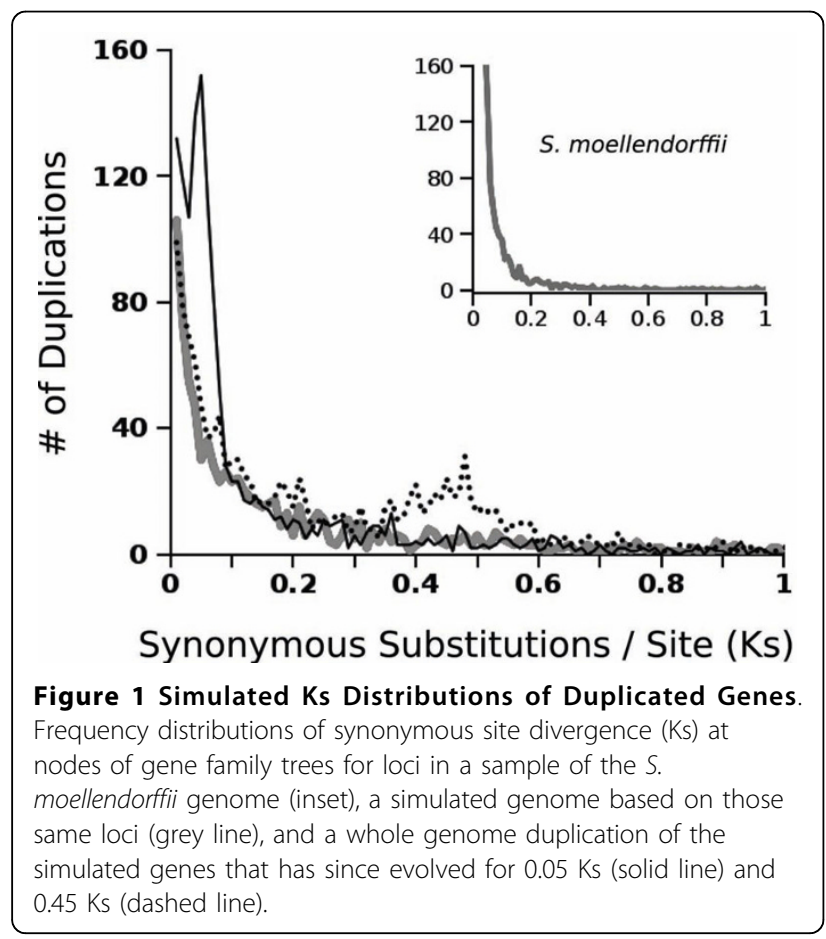

pipeline identifies sets of similar sequences as gene family members, uses alignment with known plant proteins to place sequences in reading frame, generates a gene tree for the family, and calculates the synonymous site divergence that corresponds to each node (duplication event) in the tree. Because our simulations were based on sequences from a real genome, we were able to use the existing analysis pipeline unaltered, and produce observations with the same error (e.g., in defining gene families and assigning reading frame) as occurs with real data.

Distributions of the Ks values for each duplication event are commonly used to evaluate evidence for ancient genome duplications, apparent as peaks of duplication at particular levels of divergence (a proxy for time) [15]. Detailed simulations of the sequences that yield these distributions have not been available previously, however, limiting tests of the factors generating and shaping these patterns. Histograms of duplication Ks values for our simulations (Figure 1) demonstrate that the program achieved realistic patterns of gene gain and loss, and that whole genome duplications are clearly visible as peaks that diminish with time since polyploidization. The reduction in peak prominence is due to ongoing paralog loss, and such simulations will be invaluable for generating expectations about how far back in time these events might remain observable by this method.

\section{Conclusions}

Genomic datasets offer tremendous potential to address broad evolutionary questions, but demand analytical tools that work at these same scales of biological organization. NU-IN expands the EvolSimulator platform to accommodate hypotheses involving synonymous and non-synonymous nucleotide evolution, and allows users to provide and manipulate input data as needed to address their unique needs. These features can be used to generate simulated genomic datasets under an extremely broad array of conditions affecting point mutations, copy number variation, lateral gene transfer, drift, and selection at multiple levels. Our simulation of a genome duplication event demonstrates the ability of this platform to produce realistic genome-wide patterns of gene divergence and variation from these fundamental evolutionary processes.

\section{Availability}

- Project name: NU-IN

- Project home page: http://evopipes.net/nuin.html

- Operating system(s): Linux/Unix (gcc/g++ compiler)

- Programming language: $\mathrm{C}++, \mathrm{PERL}$

- License: GNU GPL 


\section{Additional material}

Additional file 1: Example Parameters. A parameter text file used to run the NU-IN simulation program.

Additional file 2: NUIN Download 1.0.2. An archive folder (gzip tarball) of documentation and source code files for NU-IN version 1.0.2.

\section{Acknowledgements}

We thank Rob Beiko and Rob Charlebois for helpful discussions regarding the EvolSimulator program, and two reviewers for helpful comments on this manuscript. Funding was provided by the Natural Sciences and Engineering Research Council of Canada (No. 353026 to LHR).

\section{Author details}

${ }^{1}$ Department of Botany, University of British Columbia, Vancouver, BC V6T1Z4, Canada. ${ }^{2} T$ he Biodiversity Research Centre, University of British Columbia, Vancouver, BC V6T1Z4, Canada. ${ }^{3}$ Department of Biology and Center for Genomics and Bioinformatics, Indiana University, Bloomington, IN 47405, USA.

\section{Authors' contributions}

KMD, MSB, and LHR conceived of the software development. KMD wrote the software and manuscript. All authors read and approved the final manuscript.

\section{Competing interests}

The authors declare that they have no competing interests.

Received: 24 April 2010 Accepted: 2 August 2010

Published: 2 August 2010

\section{References}

1. Lynch M: The origins of genome architecture Sunderland: Sinauer Associates 2007.

2. Bomblies K, Lempe J, Epple P, Warthmann N, Lanz C, Dangl JL, Weigel D: Autoimmune response as a mechanism for a Dobzhansky-Muller-type incompatibility syndrome in plants. PLOS Biol 2007, 5:1962-1972.

3. Chapman M, Leebens-Mack J, Burke J: Positive selection and expression divergence following gene duplication in the sunflower CYCLOIDEA gene family. Mol Biol Evol 2008, 25:1260-1273.

4. Conrad DF, Pinto D, Redon R, Feuk L, Gokcumen O, Zhang Y, Aerts J, Andrews TD, Barnes C, Campbell P, Fitzgerald T, Hu M, Ihm CH, Kristiansson K, MacArthur DG, MacDonald JR, Onyiah I, Pang AWC Robson S, Stirrups K, Valsesia A, Walter K, Wei J, The Wellcome Trust Case Control Consortium, Tyler-Smith C, Carter NP, Lee C, Scherer SW, Hurles ME: Origins and functional impact of copy number variation in the human genome. Nature 2010, 464:704-712.

5. Hahn MW, Demuth JP, Han S-G: Accelerated rate of gene gain and loss in primates. Genetics 2007, 177:1949.

6. Beiko RG, Charlebois RL: A simulation test bed for hypotheses of genome evolution. Bioinformatics 2007, 23:825-831.

7. Beiko RG, Doolittle WF, Charlebois RL: The impact of reticulate evolution on genome phylogeny. Sys Biol 2008, 57:844-856.

8. Yang Z, Bielawski JP: Statistical methods for detecting molecular adaptation. Trends Ecol Evol 2000, 15:496-503.

9. PlantTribes. [http://fgp.bio.psu.edu/tribedb/index.pl].

10. Freeling $\mathrm{M}$ : Bias in plant gene content following different sorts of duplication: tandem, whole-genome, segmental, or by transposition. Ann Rev Plant Biol 2009, 60:433-453.

11. Selaginella moellendorffii v1.0. [http://genome.jgi-psf.org/Selmo1].

12. Barker MS, Kane NC, Matvienko M, Kozik A, Michelmore RW, Knapp SJ, Rieseberg LH: Multiple paleopolyploidizations during the evolution of the Compositae reveal parallel patterns of duplicate gene retention after millions of years. Mol Biol Evol 2008, 25:2445-2455.

13. Barker MS, Vogel H, Schranz ME: Paleopolyploidy in the Brassicales: analyses of the Cleome transcriptome elucidate the history of genome duplications in Arabidopsis and other Brassicales. Genome Biol Evol 2009, 1:391-399.

14. EvoPipes.net. [http://www.evopipes.net].

15. Blanc G, Wolfe KH: Widespread paleopolyploidy in model plant species inferred from age distributions of duplicate genes. Plant Cell 2004, 16:1667-1678.

doi:10.1186/1756-0500-3-217

Cite this article as: Dlugosch et al:: NU-IN: Nucleotide evolution and input module for the EvolSimulator genome simulation platform. BMC Research Notes 2010 3:217.

\section{Submit your next manuscript to BioMed Central and take full advantage of:}

- Convenient online submission

- Thorough peer review

- No space constraints or color figure charges

- Immediate publication on acceptance

- Inclusion in PubMed, CAS, Scopus and Google Scholar

- Research which is freely available for redistribution 